\title{
A New Strategy for the Preparation of Porous Silk Fibroin Scaffolds
}

\author{
Zhang $T^{1,2}$, Xiong $Q^{2}$, Shan $Y^{1 *}$ and Zhang $F^{3}$ \\ ${ }^{1}$ Department of Urology, The Second Affiliated Hospital of \\ Soochow University, Suzhou, Jiangsu, China \\ ${ }^{2}$ Department of Urology, Children's Hospital of Soochow \\ University, Suzhou, Jiangsu, China \\ ${ }^{3}$ College of Textile and Clothing Engineering, National \\ Engineering Laboratory for Modern Silk, China \\ *Corresponding author: Yuxi Shan, Department \\ of Urology, The Second Affiliated Hospital of Soochow \\ University, Suzhou, J iangsu, China
}

Received: May 29, 2021; Accepted: J une 19, 2021; Published: J une 26, 2021

\section{Introduction}

Silk is a unique material, which has historically been regarded as high-grade raw materials of textile for its strength and luster [1]. Silkbased materials have been transformed from the commodity textile to a growing web of applications in high-technology areas, especially as a biomaterial because of several desirable properties [2]. In particular, these properties include its biocompatibility, biodegradation, and versatility in processing into multiple materials formats [1]. The porous SF scaffolds have attracted considerable attention because it can provide a versatile $3 \mathrm{D}$ porous structure which is known to play a critical role for cell attachment, proliferation, migration, and tissue growth, as well as for nutrient and waste transport [3].

Porous SF scaffolds can be fabricated by a variety of methods, including lyophilization, porogens, gas foaming, etc. [1]. However, the scaffolds from pure SF solution undergo lyophilization easily form separate layers or lamellar structures rather than porous structures, and this lamellar structure will cause the loss of compressive properties and affect its application as a biomaterial [4,5]. Recently, it is reported that the porous structure of lyophilized SF scaffolds was closely related to SF assembly nanostructure. SF scaffolds with excellent pore structure could be prepared from nanofilament solution which derived from concentrated SF solution [6]. However, the way used to control drying rate of SF solution by a series of lids with hole is unstable due to the change of temperature and relative humidity around [7]. In our previous research, it was found that temperature and relative humidity played an important role in controlling SF selfassembly and the secondary structure of regenerated SF films $[7,8]$.

In this paper, we firstly prepared SF solution with nanofilaments through controlling concentrating conditions of temperature and relative humidity. Subsequently, the effect of SF nanostructure on morphology, structure and thermal property of porous SF scaffolds was investigated in detail.

\section{Material and Methods}

\section{Preparation of $B$. mori SF nanofilaments solution}

SF aqueous solution was prepared as described previously [9]
The aqueous SF solution was concentrated in Binder Temperature \& Humidity Chamber (Binder, German) at relative humidity 55\% and temperature $25^{\circ} \mathrm{C}$ for 3 days. The final concentration was $\sim 25 \mathrm{wt} \%$, determined by weighing the remaining solid after drying.

\section{Preparation of SF scaffold}

The fresh and concentrated SF solution were diluted to $2 \%$, and then were placed at $-20^{\circ} \mathrm{C}$ for $24 \mathrm{~h}$ to freeze and then lyophilized for $72 \mathrm{~h}$.

\section{Characterization}

The morphology of SF in water was observed by AFM (Veeco, Nanoscope V) in air. A $225 \mu \mathrm{m}$ long silicon cantilever with a spring constant of $3 \mathrm{Nm}-1$ was used in tapping mode at $0.5-1 \mathrm{~Hz}$ scan rate.

The morphology of SF scaffolds was observed using SEM (Hitachi S-520, Japan) at $20^{\circ} \mathrm{C}, 60 \mathrm{RH}$. Samples were mounted on a copper plate and sputter-coated with gold layer $20-30 \mathrm{~nm}$ thick prior to imaging.

The structure of the scaffolds was analyzed by FTIR on a Magna spectrometer (NicoLET5700, America), X-ray diffractometer (X'PertPro MPD, PANalytical B.V. Holland),

Thermogravimetry/differential thermal analysis (TG-DTA, PE$S^{\prime}$, America), and TA instrument Q100 DSC (TA instruments, New Castle, DE).

\section{Results and Discussion}

It is well known that pore architecture in scaffolds plays a critical role in tissue engineering for the seeded cells to form goal tissue and organs [10]. The morphology of SF in solution and porous 3D scaffolds was examined by AFM and SEM. The fresh SF solution showed a micelle structure, as Figure 1a showed, and the scaffolds from this solution showed separate layer or lamellar structure rather than porous 3D structure as our previous report (Figure 2a,2b) [5]. SF nanofilaments were formed at relative humidity $55 \%$ and $30^{\circ} \mathrm{C}$ for 3 days as the concentration increased, as Figure $1 \mathrm{~b}$ showed, and the nanofilament features of SF were similar to this observed 

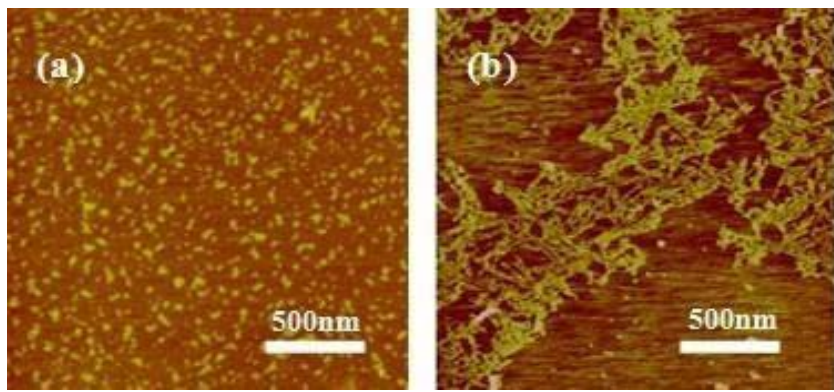

Figure 1: AFM images of SF in solution with concentration of: $0.0001 \%$. (a) The fresh SF solution; (b) The solution was treated by slowly concentrating process; (c) The SF solution isolated from the middle gland of matured $5^{\text {th }} B$. mori silkworm larvae.
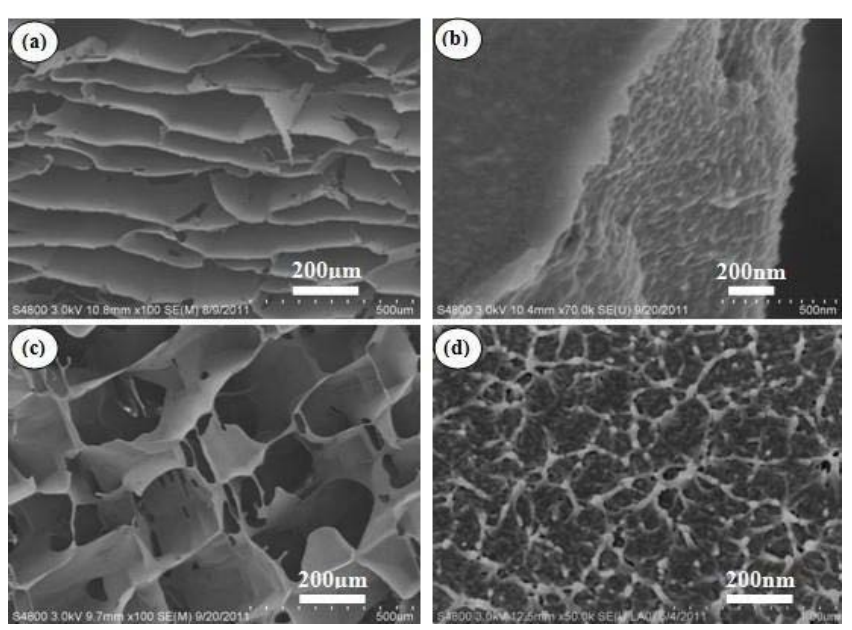

Figure 2: SEM photography of the porous SF scaffolds prepared from fresh SF solution (a) and (b); concentrated SF solution (c) and (d). Each sample is shown in two scales.

in the silk gland [11]. The scaffolds derived from above solution containing SF nanofilament demonstrated excellent porous structure, as Figure $2 c$ showed. It had been also reported that lyophilized SF scaffolds with excellent porous structure which were prepared from SF isolated from silk glands [10], indicating the important role of bionic nanofilament structure in the formation of porous structure. Furthermore, the micelle and nanofilament structure of SF observed in fresh and concentrating solution were also found in the crosssection of relevant SF scaffolds (Figure $2 b, 2 c$ ). The nanofilament, similar to ECM structure, in porous SF scaffolds would provide a favorable microenvironment for cells growth and proliferation [6].

To investigate conformational changes of SF scaffolds derived from different nanostructure solution, FTIR structural analysis was performed (Figure 3a). The peaks at $1610-1630 \mathrm{~cm}^{-1}$ and $1510-1520$ $\mathrm{cm}^{-1}$ were characteristic of silk II conformation, and the absorptions at $1648-1654 \mathrm{~cm}^{-1}$ and $1535-1542 \mathrm{~cm}^{-1}$ were correspond to silk I structure [12,13]. The scaffolds prepared with fresh SF solution (micelle) showed a strong absorption peak at $1645 \mathrm{~cm}^{-1}$ and $1532 \mathrm{~cm}$ ${ }^{1}$, corresponding to random coil, and a shoulder peak at $1518 \mathrm{~cm}^{-1}$, as shown in Figure 3a. When scaffold was formed from concentrated SF solution (nanofilament) exhibited strong absorption peak at 1652 $\mathrm{cm}^{-1}$ and $1538 \mathrm{~cm}^{-1}$ (silk I), and the shoulder peak at $1518 \mathrm{~cm}^{-1}$ (silk II)
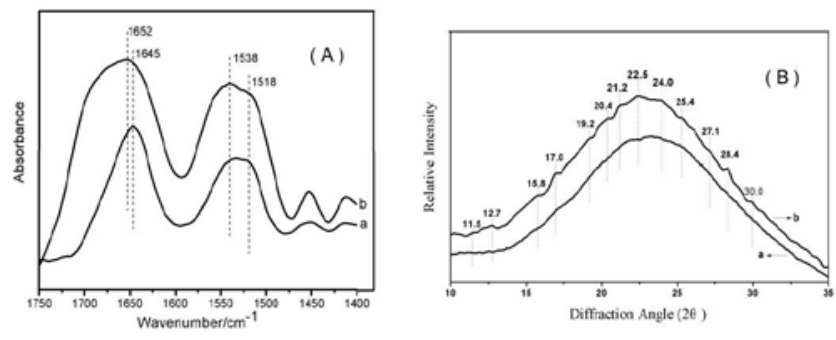

Figure 3: FT-IR spectra (A) and XRD data (B) of porous SF scaffolds prepared from (a) fresh SF solution; (b) concentrated SF solution.
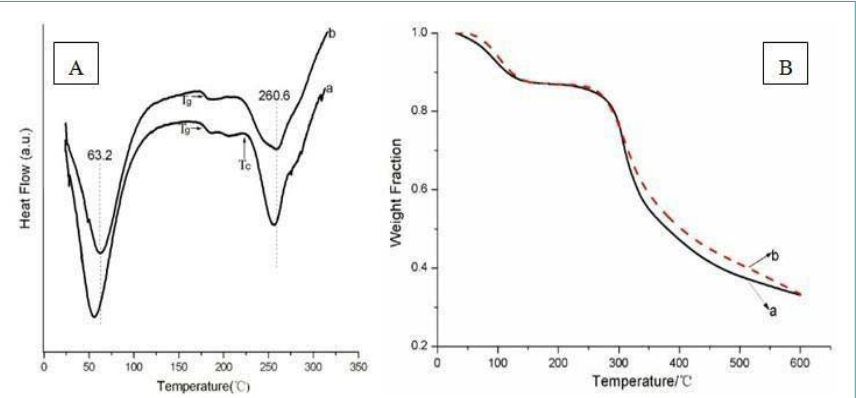

Figure 4: Standard DSC (A) and TGA curves (B) of porous SF scaffolds prepared from (a) fresh SF solution; (b) concentrated SF solution.

decreased compared to sample a, as shown in Figure 3a.

Besides FTIR, the structure changes of porous SF scaffolds were also confirmed by XRD analysis. Figure $3 \mathrm{~b}$ showed the XRD data of the SF scaffolds prepared from fresh solution (a) and concentrated solution (b). The two scaffolds showed an amorphous structure, characterized by the presence of a broad peak in the $2 \theta$ scattering angle range from 10 to $35^{\circ}$. However, compared with sample a, sample b showed diffraction peaks at $2 \theta$ values of $11.5(\mathrm{I}), 12.7(\mathrm{II})$, 19.2(I), 20.4(I), 22.5(I), 24.0(II), 25.4(I), 28.4(I), 30.0(I), indicating the formation of silk I and silk II structure, especially the formation of silk I structure [7]. The XRD results were consistent with the FTIR results, confirming the formation of silk I structure through lyophilizing the frozen concentrated SF solution. Water-insoluble silk films with silk I structure instead of random structure have been prepared by controlling the dry rate of fresh SF solution [7]. In the present study, porous SF scaffolds with partial silk I structure were prepared by concentrated SF solution.

The thermal properties of the dried SF scaffolds were also examined by standard Differential Scanning Calorimetry (DSC) and Thermal Gravimetric Analysis (TGA). Figure 4a illustrated standard DSC curves for SF scaffolds prepared from fresh (sample a) and concentrated (sample b) SF solution. Sample a showed an endothermic peak at $56^{\circ} \mathrm{C}$, an obvious glass transition Temperature (Tg) at around $180^{\circ} \mathrm{C}$, a non-isothermal crystallization peak appeared around $230^{\circ} \mathrm{C}$, and a degradation peak at $257^{\circ} \mathrm{C}$. The endothermic peak at $56^{\circ} \mathrm{C}$ was due to the evaporation of bound water. The glass transition temperature regions of sample a was similar to previous report [14]. After the glass transition temperature regions, an obvious nonisothermal crystallization peak at $230^{\circ} \mathrm{C}$ is related to the thermal transition of SF molecular from unstable non-crystal structure to crystal structure [15]. Finally, sample a started to degrade with 
endothermic peak at around $257^{\circ} \mathrm{C}$.

Sample b showed a $\mathrm{Tg}$ at around $180^{\circ} \mathrm{C}$ which was similar to sample a, but the crystallization peak disappeared because of the formation of silk I crystals which had been confirmed above by FTIR and XRD. The disappear of crystallization peak indicated that silk I was a stable structure under degradation peak at around $260^{\circ} \mathrm{C}$. The endothermic peak of water inside SF scaffolds and degradation peak increased to 63.2 and $260.6^{\circ} \mathrm{C}$, which were likely due to the silk I structure formed during the concentrated process and the silk I crystal is a hydrated structure [7].

The TGA results confirmed that stronger water-silk interactions and more stable thermal property due to the increase of silk I content formed during concentrated process, though the weight loss was same when the temperature reached $120^{\circ} \mathrm{C}$ and $600^{\circ} \mathrm{C}$, as Figure $4 \mathrm{~b}$ showed.

\section{Conclusions}

In this paper, we prepared SF scaffolds with 3D porous structure by controlling the SF assembly. Under relative humidity $55 \%$ and $30^{\circ} \mathrm{C}$, SF assembled nanofilaments after 2 days with the concentration increase from about $2 \mathrm{wt} \%$ to $30 \mathrm{wt} \%$. SF scaffolds derived from nanofilament solution demonstrated porous and silk I structure, in contrary, SF scaffolds derived from micelles solution showed layered and random coil structure, suggesting that the biotic structure of SF nanofilament is a key parameter for controlling the morphology and structure of regenerated SF scaffolds. SF scaffolds with porous structure would further facilitate the use of SF in tissue engineering.

\section{Acknowledgement}

This work was supported financially by Nature Science Foundation of Jiangsu Province (SBK2020041082, BK20191168) and Suzhou Planning Project of Science and Technology (SYS2019005, SYS2018052).

\section{References}

1. Rockwood DN, Preda RC, Yucel T, Wang X, Lovett ML, Kaplan DL. Rockwood DN, Preda RC, Yucel T, Wang X, Lovett ML, Kaplan DL. Nat Protoc. 2011; 6 1612-1631. Nat Protoc. 2011; 6: 1612-1631.
2. Omenetto FG, Kaplan DL. Science. New Opportunities for an Ancient Material. 2010; 329: 528-531.

3. Vepari C, Kaplan DL. Prog Polym Sci. Silk as a Biomaterial. 2007; 32: 991 1007.

4. Lv Q, Feng Q, Hu K, Cui F. Polymer. Three-dimensional fibroin/collagen scaffolds derived from aqueous solution and the use for HepG2 culture. 2005; 46: $12662-12669$

5. Lu Q, Zhang X, Hu X, Kaplan DL. Macromol Biosci. Green process to prepare silk fibroin/gelatin biomaterial scaffolds. 2010; 10: 289-298.

6. Lu Q, Wang X, Lu S, Li M, Kaplan DL, Zhu H. Nanofibrous architecture of silk fibroin scaffolds prepared with a mild self-assembly process. Biomaterials. 2011; 32: 1059-1067.

7. Lu Q, Hu X, Wang X, Kluge JA, Lu S, Cebe P, et al. Water-insoluble silk films with silk I structure. Acta Biomater. 2010; 6: 1380-1387.

8. Ming J, Zuo B. Silk I structure formation through silk fibroin self-assembly. $J$ Appl Polym Sci. 2012; 125: 2148-2154.

9. Zhang F, Zuo B, Fan Z, Xie Z, Lu Q, Zhang X, et al. Mechanisms and contro of silk-based electrospinning. Biomacromolecules. 2012; 13: 798-804.

10. Mandal BB, Kundu SC. Cell proliferation and migration in silk fibroin 3D scaffolds. Biomaterials. 2009; 30: 2956-2965

11. Lu Q, Zhu H, Zhang C, Zhang F, Zhang B, Kaplan DL. Silk SelfAssembly Mechanisms and Control From Thermodynamics to Kinetics. Biomacromolecules. 2012; 13: 826-832.

12. Hu X, Shmelev K, Sun L, Gil ES, Park SH, Cebe P, et al. Regulation of silk material structure by temperature-controlled water vapor annealing. Biomacromolecules. 2011; 12: 1686-1696.

13. Jin HJ, Park J, Karageorgiou V, Kim UJ, Valluzzi R, Cebe P, et al. Adv Funct Mater. 2005; 15: 1241-1247

14. Hu X, Kaplan DL, Cebe P. Dynamic Protein-Water Relationships during $\beta$-Sheet Formation. Macromolecules. 2008; 41: 3939-3948.

15. Hu X, Lu Q, Sun L, Cebe P, Wang X, Zhang X, et al. Biomacromolecules. 2019. 